\title{
Photographic variability survey in the M42 region
}

\author{
Luiz Paulo R. Vaz ${ }^{1}$, Gustavo H. R. A. Lima ${ }^{1}$ and Bo Reipurth ${ }^{2}$ \\ ${ }^{1}$ Departamento de Física - ICEx - UFMG - Brazil \\ emails: lpv@fisica.ufmg.br, styx@fisica.ufmg.br \\ ${ }^{2}$ IfA - Univ. of Hawaii - USA \\ email: reipurth@ifa.hawaii.edu
}

\begin{abstract}
In order to detect variable stars in the well known star forming region, the Orion Nebula Cluster, a series of 22 exposures taken from November 1996 to October 1998, using the ESO 100/152cm Schmidt telescope, covering a field of $5^{\circ} \times 5^{\circ}$ was analyzed. The films (Kodak Tech-Pan 4415 emulsions, effective spectral range from $\sim 630 \mathrm{~nm}$ to $690 \mathrm{~nm}$ ) were digitized by the SuperCOSMOS machine, the measurements calibrated to the $\mathrm{R}$ magnitude of the USNO B1.0 catalogue and differential photometry was performed throughout the whole field. In the process, a set of 260 stars that remained constant in the 22 films and were well distributed over the field was selected and used as comparison stars for the differential photometry of all the other stars in the field. Diverse statistical studies were performed in order to characterize the type and degree of variability of the objects. The 22 films, all exposed for 30 minutes each, were stacked together at our request by the SuperCOSMOS team, producing perhaps the deepest wide field image of M42 ever taken.

This database ( $>150000$ objects, mostly stars and $\sim 2 \%$ galaxies) is going to be used as a starting point for the Variable Young Stellar Object Survey (VYSOS) project, which consists of 2 fully automated robotic telescopes of $41 \mathrm{~cm}$ each, one installed on Mauna Loa (Hawaii, USA) and the other at Cerro Armazones (Chile), both using the Pan STARRS set of photometric filters. We discovered thousands of new variable stars within the $5^{\circ} \times 5^{\circ}$ region studied. We have variability statistics for all objects and are classifying the variable stars according to the variability type and amplitude. We intend to make this database available via the WEB.
\end{abstract}

Keywords. stars: variables, stars: pre-main sequence, stars: formation, astronomical data bases, surveys, open clusters and associations: individual (M42)

\section{Acknowledgements}

We are very grateful to Dr. Harvey MacGillivray of the SuperCosmos center at the Royal Observatory Edinburgh. We also thank Dr. Sue Tritton as head of the Wide Field Astronomy Unit for the allocation of time on the SuperCoSMOS machine. We acknowledge use of the General Catalogue of Variable Stars maintained by the Sternberg Astronomical Observatory, and thank Dr. Nikolai Samus for providing early release of data on variables in Orion. LPV and GHRAL gratefully acknowledge partial support from the Brazilian Agencies CNPq, CAPES, FAPEMIG. This publication makes use of data products from 2MASS, a joint project of the Univ. of Massachusetts and IPAC/California Institute of Technology, funded by NASA and NSF. This research has made use of the USNOFS Image and Catalogue Archive operated by the United States Naval Observatory, Flagstaff Station, of the SIMBAD database, operated at CDS, Strasbourg, France, and of NASA's Astrophysical Data System bibliographic services. 